\title{
Notificações, internações e mortes por lesões autoprovocadas em crianças nos sistemas nacionais de saúde do Brasil
}

\author{
Notifications, hospitalizations and deaths from self-harm \\ in children in Brazil's national health systems
}

Joviana Quintes Avanci (https://orcid.org/0000-0001-7779-3991) ${ }^{1}$

Liana Wernersbach Pinto (https://orcid.org/0000-0003-1928-9265) ${ }^{1}$

Simone Gonçalves de Assis (http://orcid.org/0000-0001-5460-6153) ${ }^{1}$

${ }^{1}$ Departamento de Estudos sobre Violência e Saúde Jorge Careli (Claves), Escola Nacional de Saúde Pública - Fundação Oswaldo Cruz. R. Leopoldo Bulhões 1480, Manguinhos. 21041-210 Rio de Janeiro RJ Brasil.

avanci@globo.com

\begin{abstract}
There are virtually no statistics on childhood suicidal behavior globally. This setting can be partly explained by the social representation of joy in this early phase of life. This paper aims to analyze information on self-harm reports, hospitalizations, and deaths among children aged 5-9 years in Brazil to know its magnitude and distribution from 2006 to 2017, based on data from national health information systems. The results show 58 deaths of Brazilian children with intentional self-harm, primarily male, white, and nine years old. Hanging was the most commonly used mechanism for children to kill themselves. A total of 1,994 hospitalizations were recorded for suicide attempts in children in the period 2006-2017, with a predominance of males in all regions. Regarding notifications, most refer to children between 8 and 9 years of age, black and female, emphasizing self-poisoning. Evidence that any suicidal behavior in childhood is strongly associated with attempted or completed suicide in adolescence and adulthood is one of the main indications of the need to prevent this behavior in the first decade of life. Key words Child, Suicidal behavior, Suicide attempt, Suicide
\end{abstract}

Resumo Praticamente não há estatísticas sobre o comportamento suicida na infância no mundo. Em parte, este cenário pode ser explicado pela representação social de alegria desta fase da vida. Este artigo tem como objetivo analisar informações sobre notificações, internações e mortes por lesões autoprovocadas entre crianças de cinco a nove anos no Brasil, visando conhecer sua magnitude e distribuição no período de 2006 a 2017, a partir dos dados dos sistemas nacionais de informação de saúde. Foram identificados 58 óbitos de crianças brasileiras decorrentes dessa causa, com a maioria sendo do sexo masculino, de cor da pele branca e com nove anos de idade. O enforcamento foi o meio mais utilizado pelas crianças para se matar. As internações por tentativas de suicídio no período de 2006-2017 somaram 1.994 casos, com predominância entre os meninos em todas as regiões. No que diz respeito às notificações, a maioria se refere a crianças entre oito e nove anos de idade, com cor da pele parda e do sexo feminino, com destaque à autointoxicação. A evidência de que qualquer comportamento suicida na infância está fortemente associado às tentativas ou ao suicídio consumado na adolescência e na vida adulta é uma das principais indicações da necessidade de prevenção desse comportamento na primeira década da vida.

Palavras-chave Criança, Comportamento suicida, Tentativa de suicídio, Suicídio 


\section{Introdução}

O comportamento de se ferir, de tentar se matar ou de acabar com a própria vida é pouco usual na infância e um tema ainda incipiente na literatura nacional e internacional. Está imerso em tabus culturais e cercado por indagações e desconhecimento, inclusive entre estudiosos e profissionais que atuam junto à população infantil ${ }^{1,2}$. Embora raro em sua magnitude, não é um fenômeno excepcional, havendo sub-registro de sua ocorrência por causa, em parte, da própria representação social da infância, marcada pela ludicidade e alegria, com o desejo de morrer ou de um sofrimento demasiado parecendo incompatível com essa fase da vida ${ }^{3}$.

É grande a dificuldade de delimitar conceitualmente a lesão autoprovocada e o comportamento suicida, especialmente na infância, devido à falta, na maioria das vezes, de evidência quanto à intencionalidade do ato. Sob uma perspectiva conceitual ampliada, a Organização Mundial de Saúde ${ }^{4}$ denomina a lesão autoprovocada como uma violência que a pessoa inflige contra si mesma, sendo classificada como comportamento suicida e como autoagressão (engloba arranhaduras, cortes e mordidas em si própria, além de amputação de membros). O comportamento suicida é caracterizado por todo ato pelo qual o indivíduo causa lesão a si mesmo, e contempla desde ideações e planejamentos até as tentativas e o suicídio consumado ${ }^{5}$. De maneira similar, a Classificação Estatística Internacional de Doenças e Problemas Relacionados à Saúde (CID10) considera como autoprovocados as lesões e os envenenamentos intencionalmente infligidos pela pessoa a si própria e as tentativas de suicídio 6 . Portanto, subjacente a esse debate está o alívio do sofrimento, que na maioria dos casos é a principal função do ato de se ferir ou se matar.

Outra barreira para a compreensão do fenômeno na infância está vinculada à definição do momento em que o desenvolvimento cognitivo do conceito de morte se instaura na criança, o que tende a ocorrer em torno dos nove anos de idade, com diferenciações em função da convivência com a morte, do histórico de vida familiar e do processo de elaboração sobre o tema a partir de histórias, desenhos animados e contos de fadas ${ }^{1,7}$. Nas etapas anteriores do desenvolvimento infantil, há ideias limitadas sobre a morte, com a crença na reversibilidade e o temor da morte da genitora, mas não de si mesmo. A definição do comportamento suicida tem como pré-requisito a consciência da finitude da vida, inclusive de sua própria ${ }^{1}$. Todavia, Pedroso ${ }^{2}$ esclarece que o fato de a criança ter um conceito da morte diferente dos adultos não significa necessariamente que ela não poderá atentar contra a sua própria vida. Fensterseifer e Werlang ${ }^{3}$ alertam sobre a necessidade de se romper com o mito da infância, desfazendo a concepção de que uma criança é incapaz de atentar intencionalmente contra a própria vida, uma vez que elas têm condições para se ferir, planejar e realizar um suicídio com sucesso. Esses autores explicam ainda que cerca de metade dos acidentes envolvendo crianças pode ser de tentativas mascaradas de suicídio.

Em todo o mundo, praticamente não há estatísticas sobre o fenômeno entre crianças até nove anos de idade, e quando existem costumam ser subestimadas. No início dos anos 2000, Beautrais ${ }^{8}$ já alertava que as estimativas internacionais sugeriam taxas de suicídio para crianças de 5 a 14 anos de cerca de uma a duas mortes por $100.000^{9}$. Apesar de baixas e bem inferiores às da adolescência, há uma tendência de aumento da taxa de suicídio em crianças com menos de 15 anos em vários países ${ }^{10-12}$. Em 2015, o Centers for Disease Control and Prevention ${ }^{13,14}$ mostrou que o suicídio foi a sexta principal causa de morte entre crianças de 5 a 12 anos de idade nos Estados Unidos, com ligeiro aumento entre os anos de $2013 \mathrm{e}$ 2014 (cerca de 16\%). Nos EUA o suicídio é a terceira principal causa de morte entre adolescentes de 10 a 14 anos de idade ${ }^{15}$, e em todo o mundo, assim como no Brasil, permanece no topo entre adolescentes mais velhos ${ }^{16}$.

A relevância de investigar a lesão autoprovocada na infância se consolida na tendência do continuum de severidade ao longo do curso do desenvolvimento, que pode se iniciar com atos de se ferir e evoluir para a ideação, o planejamento, a tentativa e evoluir até o suicídio consumado ${ }^{17,18}$, o que não significa que este percurso é sempre rigorosamente seguido. $\mathrm{O}$ ato de se ferir sem evidência de intenção suicida é um fenômeno típico da adolescência e tende a decrescer na vida adul$\mathrm{ta}^{19}$. A ideação suicida em pré-adolescentes, por sua vez, tem sido consistentemente associada às tentativas em adolescentes ${ }^{18-20}$. Brent ${ }^{21}$ alerta para a necessidade de compreender melhor o comportamento suicida em faixas etárias mais novas, entendendo os antecedentes e os perfis das vítimas cada vez mais cedo para que se possa detectar e intervir precocemente.

Uma série de fatores está associada à gênese do fenômeno. Nos anos 1980, Kosky ${ }^{22}$, estudando crianças australianas que tentaram o suicídio, concluiu que a maioria era do sexo masculino, 
usava métodos violentos para tentar morrer, tinha fraco desempenho acadêmico e havia sido exposta a circunstâncias familiares difíceis, incluindo violência intrafamiliar, separação parental e divórcio. Entre os principais fatores de risco tradicionalmente associados ao comportamento suicida na infância estão: transtorno de humor, privação emocional, perda de pessoas significativas, abuso físico, abuso de álcool e outras drogas pelos pais, histórico familiar de suicídio e acesso a métodos usados para cometer suicídio ${ }^{23-25}$. A impulsividade é o fator individual chave para aqueles com maior risco para o suicídio, devido ao difícil processamento cognitivo durante o ato ${ }^{23}$.

O conhecimento sobre a lesão autoprovocada na infância é muito escasso e a maior parte dos trabalhos se baseia em estudos de caso, em amostras não representativas, prioriza o período da adolescência e aborda o problema em uma ampla faixa etária de 5 a 14 anos, o que é inadequado face às significativas diferenças desenvolvimentais, o que dificulta generalizações ${ }^{20}$.

Embora o número absoluto da lesões autoprovocadas possa ser pequeno em termos quantitativos frente ao tamanho da população, conhecer sua epidemiologia certamente dá visibilidade ao fenômeno e apoia o conhecimento sobre as características das crianças que se autolesionam, tentam ou morrem dessa maneira, portanto possibilita atuar na prevenção e em decisões do setor saúde. Este artigo busca analisar a magnitude e a distribuição das notificações, internações e mortes por lesões autoprovocadas em crianças de cinco a nove anos ocorridos no Brasil no período de 2006 a 2017, a partir dos dados dos sistemas nacionais de informação de saúde.

\section{Métodos}

Trata-se de um estudo transversal sobre as lesões autoprovocadas em crianças brasileiras entre cinco a nove anos de idade no período de 2006 a 2017, sendo empregados dados secundários de três sistemas nacionais de informação de saúde.

Para a análise das notificações sobre lesões autoprovocadas em crianças no período de 2011 a 2017, baseou-se no Sistema de Informação de Agravos de Notificação (SINAN) de violência doméstica, sexual e outras, cujos dados são obtidos a partir do preenchimento da Ficha de Notificação de Violência Interpessoal/Autoprovocada para todo caso suspeito ou confirmado de violência atendido em serviços de saúde públicos e privados do país. Este sistema de vigilância é consti- tuído por dois componentes, porém neste artigo apenas o componente do SINAN/VIVA contínuo é analisado. É um sistema recente e apresenta fragilidades, pois apenas em 2011 a notificação de violência foi inserida na lista de eventos de notificação compulsóriaa ${ }^{26,27}$. Além disso, até 2014 o sistema de informação não permitia o registro da lesão autoprovocada para pessoas com idade inferior a 14 anos.

A primeira etapa da análise consistiu na verificação da evolução temporal das notificações por violências, sem distinção de faixa etária e entre crianças de cinco a nove anos de idade, e também o número de notificações classificadas como lesão autoprovocada em crianças da mesma faixa etária a partir dos códigos da CID-10 (X60-X84) para o período de 2011 a 2017. Os códigos X60-X84 se referem a lesões autoprovocadas intencionalmente por: (X60) autointoxicação por/exposição intencional a substâncias farmacológicas (analgésicos, antipiréticos e anti-reumáticos, não-opiáceos, drogas anticonvulsivantes sedativos, narcóticos), álcool, solventes orgânicos, pesticidas; (X70) enforcamento, estrangulamento, sufocação, afogamento e submersão, disparo de arma de fogo de mão, dispositivos explosivos, fumaça, fogo e chamas, vapor d'água, gases ou objetos quentes, objeto cortante ou penetrante, objeto contundente; e (X80) por precipitação de um lugar elevado, precipitação ou permanência diante de um objeto em movimento, impacto de um veículo a motor e por meios não especificados ${ }^{6}$.

Posteriormente, realizou-se a análise bivariada dos eventos segundo algumas características das crianças notificadas (sexo, idade, cor da pele, local de ocorrência e meio empregado) no período de 2015 a 2017, os quais apresentam dados mais qualificados para a faixa etária. Para essa segunda etapa, os dados analisados advêm das notificações em que foi assinalada a opção "sim" no campo "lesão autoprovocada" e empregados os códigos X60 a X84 da CID-10 no campo "circunstância da lesão". De forma complementar, verificou-se ainda os dados do campo "tipo de violência - outros" (campo aberto para digitação). Em algumas situações (quatro casos), observou-se que esse campo havia sido preenchido com os seguintes termos: "suicídio", "tentativa de suicídio" e "autoinflingida”, mas que contraditoriamente se assinalou a opção "não" no campo "lesão autoprovocada". Considerou-se então que houve erro de preenchimento e tais casos foram incluídos na análise realizada no artigo.

Para a apreciação das internações por tentativas de suicídio, o Sistema de Informações 
Hospitalares (SIH) serviu como fonte dos dados. Esse sistema registra informações sobre a morbidade por meio dos formulários da Autorização de Internação Hospitalar, com finalidade de efetuar o pagamento dos procedimentos realizados durante as internações em hospitais públicos e conveniados ao Sistema Único de Saúde. Os pontos negativos desse sistema são: o excesso de informações sobre procedimentos caros, cobranças por intervenções não efetuadas e realização de exames desnecessários, e a não cobertura dos atendimentos ocorridos em emergências ou prontos-socorros, que são locais privilegiados para a chegada dos casos de tentativa de suicídio.

Para o estudo das tentativas de suicídio no SIH (DATASUS) foram empregados os mesmos códigos da CID-10 usados para as notificações (X60-X84). Os dados foram analisados em dois intervalos de tempo: 2006-2011 e 2012-2017. A descrição analítica da internação por tentativa de suicídio se deu por região e unidade da federação (UF) de acordo com as seguintes variáveis: sexo, cor da pele, média do tempo de permanência e óbito do paciente. Também foram calculadas a evolução das taxas de internação (por $100 \mathrm{mil}$ habitantes) segundo regiões do país e entre os sexos para o período de 2006 a 2017. Para esse cálculo, o número de internações classificadas nos códigos X60 a X84 da CID-10 compuseram o numerador, e a população brasileira para a faixa etária de cinco a nove anos (por sexo e região), o denominador. Deve-se destacar, contudo, que esse é um cálculo aproximado, visto que o número de internações não corresponde ao de pessoas, uma vez que uma pessoa pode ter mais de uma internação no período.

Para a análise do óbito por suicídio na faixa etária de cinco a nove anos, utilizou-se o Sistema de Informações sobre Mortalidade (SIM), em que causa mortis é atestada pelo médico no atestado de óbito (DATASUS). Também aqui os códigos X60-X84 (lesões autoprovocadas intencionalmente) da CID-10 foram empregados. $\mathrm{Na}$ seleção dos óbitos a serem analisados, utilizouse a variável circunstância do óbito, que indica a provável circunstância de morte não natural, tendo como possibilidade de resposta os seguintes códigos: 1 - acidente; 2 - suicídio; 3 - homicídio; 4 - outro; e 9 - ignorado. A análise da mortalidade foi feita a partir da descrição da distribuição dos óbitos por suicídio segundo o sexo da criança para as seguintes variáveis: ano, idade, cor da pele, escolaridade, local de ocorrência do óbito, unidade da federação (UF), região, meio empregado (categoria CID-10) e fonte da informação.
Em função das baixas contagens apresentadas para o evento sob análise, foram utilizadas as frequências absolutas e relativas em detrimento das taxas.

\section{Resultados}

Na Tabela 1 é apresentada a evolução das notificações por violências em todas as faixas etárias, evidenciando as que ocorrem entre crianças de cinco a nove anos que chegaram aos serviços de saúde no período de 2011 a 2017 e destacando a frequência da lesão autoprovocada nas idades estudadas. Como se pode observar, as notificações cresceram de forma expressiva ao longo dos anos, seja para todas as formas de violências, seja para a lesão autoprovocada. No período de 2011 a 2017, houve 196 notificações por lesão autoprovocada em crianças de cinco a nove anos. É importante destacar que, embora haja registros, até 2014 o sistema não permitia a atribuição de lesões autoprovocadas para menores de 14 anos.

$\mathrm{Na}$ Tabela 2 podem ser observadas as características das crianças de cinco a nove anos de idade notificadas por lesão autoprovocada no SINAN/ VIVA contínuo. É importante lembrar que chegou-se a esse número de notificações a partir do uso concomitante de duas variáveis disponíveis na ficha de notificação, a saber: (1) lesão autoprovocada ("sim") e circunstância da lesão (códigos X60 a X84). A esses acrescentaram-se quatro casos em que se assinalou a opção "não" em lesão autoprovocada, porém interpretamos como erro de preenchimento, visto que no campo "tipo de violência - outro" foi apontado que se tratava de um suicídio ou tentativa.

De 2015 a 2017, o perfil das 78 notificações de crianças atendidas pelos serviços de saúde por lesão autoprovocada mostra que a maioria $(56,4 \%)$ ocorreu no ano de 2017; são crianças com oito e nove anos de idade (62,8\%); têm cor da pele branca e $(46,2 \%)$ e parda/preta $(39,7 \%)$ - apenas uma era indígena - e são do sexo feminino. Em relação ao local de ocorrência, 80,8\% ocorreram na própria residência e na grande maioria o evento foi ocasionado por autointoxicação $(54 \%)$, seguida por objeto contundente, cortante ou penetrante $(24,3 \%)$ e, de maneira decrescente, por enforcamento, precipitação de um lugar elevado, impacto de um veículo a motor e outros meios.

Os dados referentes às internações por tentativas de suicídio podem ser observados na Tabela 3 . 
Tabela 1. Distribuição das notificações por violências e lesões autoprovocadas em crianças de cinco a nove anos de idade (SINAN/VIVA contínuo), 2011-2017, Brasil.

\begin{tabular}{lcccc}
\hline Ano & $\begin{array}{c}\text { Total de } \\
\text { notificações por } \\
\text { violência em todas } \\
\text { faixas etárias }\end{array}$ & $\begin{array}{c}\text { Total de notificações por violência } \\
\text { entre crianças de cinco a nove anos }\end{array}$ & $\begin{array}{c}\text { Eventos classificados } \\
\text { nos códigos de lesões } \\
\text { autoprovocadas (X60-X84) } \\
\text { - cinco a nove anos }\end{array}$ \\
\hline 2011 & 107.530 & $\mathbf{N}$ & $\%$ & 15 \\
2012 & 157033 & 7.048 & 6,6 & 20 \\
2013 & 188.728 & 10.292 & 9,6 & 23 \\
2014 & 201.044 & 11.227 & 10,4 & 15 \\
2015 & 227.852 & 12.561 & 10,9 & 35 \\
2016 & 243.192 & 13.741 & 11,7 & 26 \\
2017 & 307.307 & 16.039 & 12,8 & 62 \\
\hline
\end{tabular}

Fonte: Sistema de Informação de Agravos de Notificação (SINAN), Ministério da Saúde.

Tabela 2. Características das crianças de cinco a nove anos de idade com notificações de lesões autoprovocadas (SINAN/VIVA contínuo) segundo sexo, 2015-2017, Brasil.

\begin{tabular}{|c|c|c|c|c|c|c|}
\hline \multirow{2}{*}{ Variável } & \multicolumn{2}{|c|}{ Feminino $(\mathrm{N}=40)$} & \multicolumn{2}{|c|}{ Masculino $(\mathrm{N}=38)$} & \multicolumn{2}{|c|}{ Total } \\
\hline & $\mathbf{n}$ & $\%$ & $\mathbf{n}$ & $\%$ & $\mathbf{n}$ & $\%$ \\
\hline \multicolumn{7}{|l|}{ Idade } \\
\hline 5 & 5 & 12,5 & 4 & 10,5 & 9 & 11,5 \\
\hline 6 & 5 & 12,5 & 3 & 7,9 & 8 & 10,3 \\
\hline 7 & 4 & 10,0 & 8 & 21,1 & 12 & 15,4 \\
\hline 8 & 9 & 22,5 & 11 & 28,9 & 20 & 25,6 \\
\hline 9 & 17 & 42,5 & 12 & 31,6 & 29 & 37,2 \\
\hline \multicolumn{7}{|l|}{ Cor da pele } \\
\hline Branca & 17 & 42,5 & 19 & 50,0 & 36 & 46,2 \\
\hline Preta & 2 & 5,0 & 1 & 2,6 & 3 & 3,8 \\
\hline Parda & 12 & 30,0 & 16 & 42,1 & 28 & 35,9 \\
\hline Indígena & 1 & 2,5 & 0 & 0,0 & 1 & 1,3 \\
\hline Ignorado & 8 & 20,0 & 2 & 5,3 & 10 & 12,8 \\
\hline \multicolumn{7}{|l|}{ Local de ocorrência } \\
\hline Residência & 34 & 85,0 & 29 & 76,3 & 63 & 80,8 \\
\hline Habitação coletiva & 0 & 0,0 & 2 & 5,3 & 2 & 2,6 \\
\hline Escola & 3 & 7,5 & 4 & 10,5 & 7 & 9,0 \\
\hline Outro & 1 & 2,5 & 1 & 2,6 & 2 & 2,6 \\
\hline Ignorado & 2 & 5,0 & 2 & 5,3 & 4 & 5,1 \\
\hline \multicolumn{7}{|l|}{ Meio empregado } \\
\hline X60 & 0 & 0,0 & 1 & 2,6 & 1 & 1,3 \\
\hline X61 & 4 & 10,0 & 0 & 0,0 & 4 & 5,1 \\
\hline $\mathrm{X} 62$ & 14 & 35,0 & 4 & 10,5 & 18 & 23,1 \\
\hline X64 & 1 & 2,5 & 0 & 0,0 & 1 & 1,3 \\
\hline X66 & 5 & 12,5 & 7 & 18,4 & 12 & 15,4 \\
\hline X68 & 2 & 5,0 & 0 & 0,0 & 2 & 2,6 \\
\hline X69 & 1 & 2,5 & 3 & 7,9 & 4 & 5,1 \\
\hline $\mathrm{X} 70$ & 1 & 2,5 & 10 & 26,3 & 11 & 14,1 \\
\hline $\mathrm{X} 78$ & 0 & 0,0 & 1 & 2,6 & 1 & 1,3 \\
\hline $\mathrm{X} 79$ & 1 & 2,5 & 0 & 0,0 & 1 & 1,3 \\
\hline $\mathrm{X} 80$ & 10 & 25,0 & 8 & 21,1 & 18 & 23,1 \\
\hline X82 & 1 & 2,5 & 0 & 0,0 & 1 & 1,3 \\
\hline $\mathrm{X} 83$ & 0 & 0,0 & 4 & 10,5 & 4 & 5,1 \\
\hline
\end{tabular}

Fonte: Sistema de Informação de Agravos de Notificação (SINAN), Ministério da Saúde. 


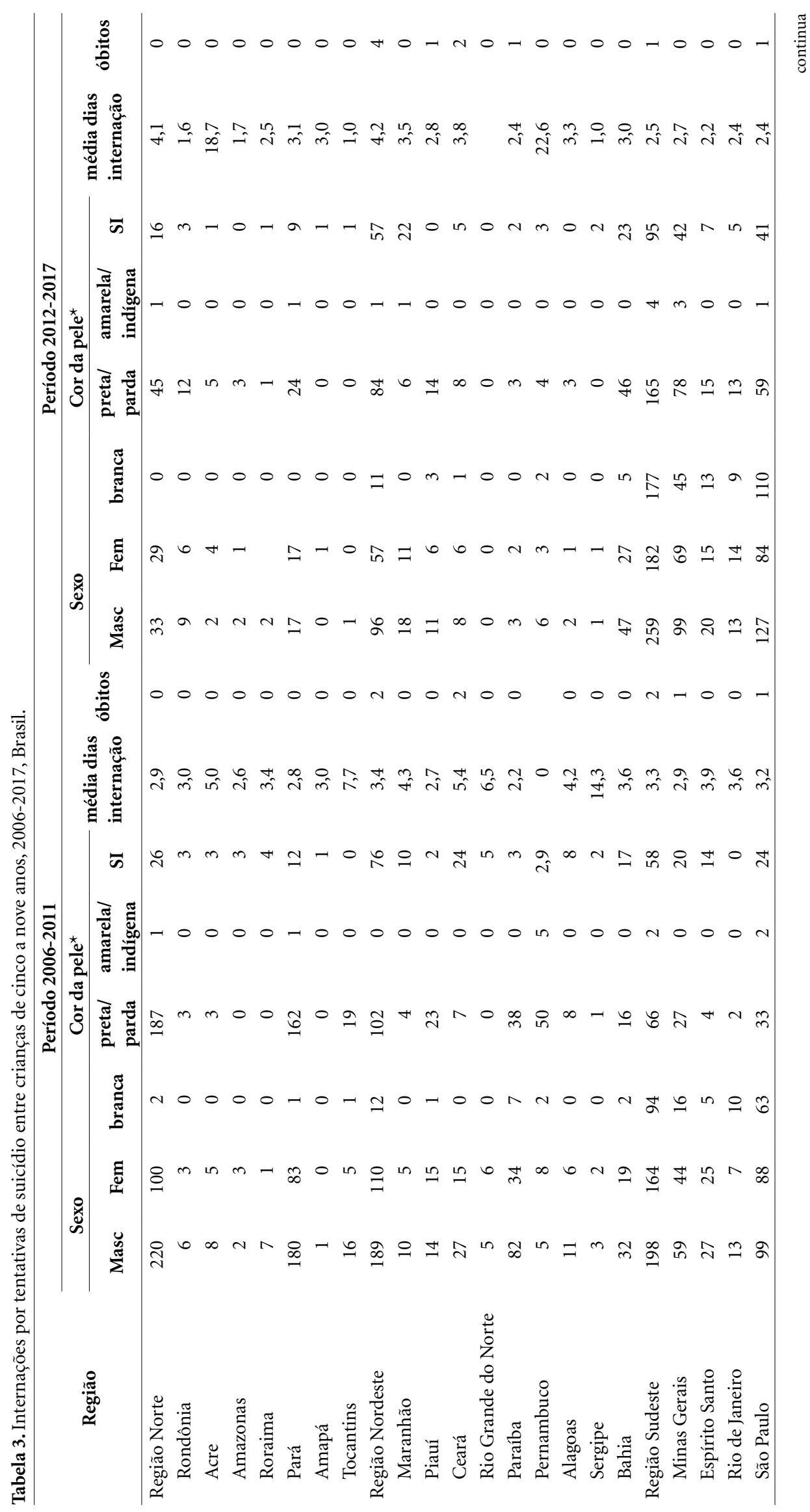




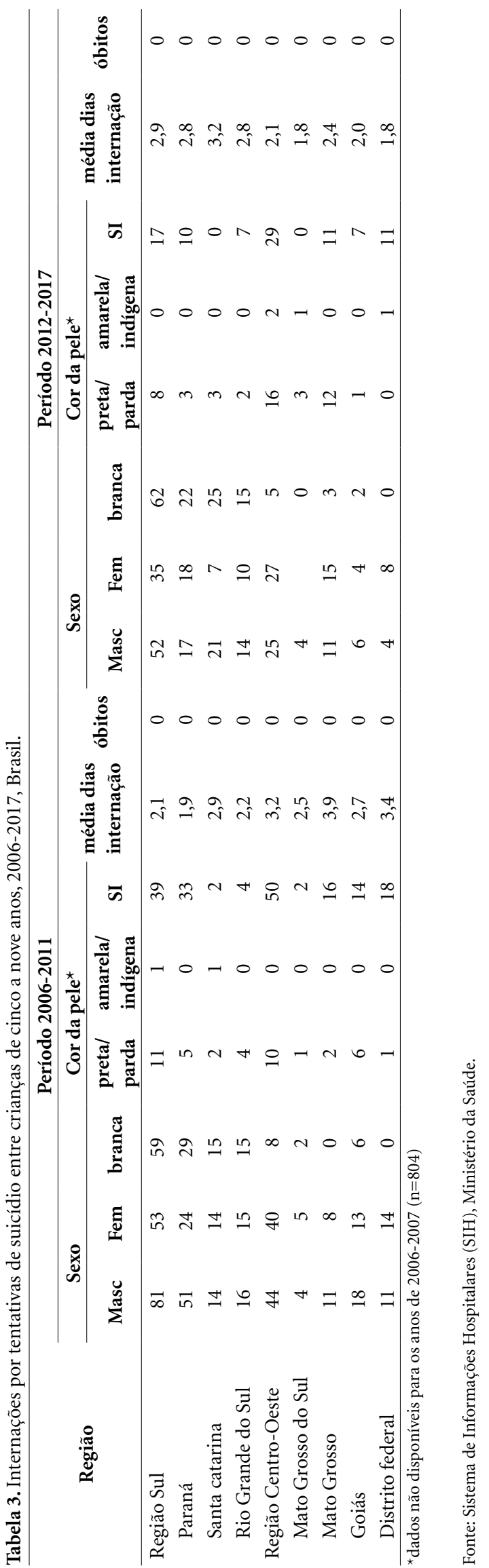

No período de 2006 a 2011 ocorreram 1.199 internações por tentativa de suicídio em crianças, com predominância nas regiões Norte e Sudeste. Entre as UFs, destacam-se os dados de Pará, Paraíba, Minas Gerais e São Paulo. Verifica-se que no período estudado há predominância de internações de indivíduos do sexo masculino para todas as regiões. A cor da pele branca é destaque nas regiões Sudeste e Sul, e a cor parda nas regiões Norte, Nordeste e Centro-Oeste. Os casos geraram internações curtas, com máximo de seis dias, à exceção do estado do Sergipe, onde a média de dias de permanência na unidade hospitalar foi de 14,3 dias. Ocorreram oito óbitos entre as 1.199 internações analisadas.

Para o período seguinte (2012-2017), foram constatadas 795 internações, novamente com predomínio masculino. Contudo, no Acre, Rio de Janeiro, Paraná, Mato Grosso e Distrito Federal, as internações foram maiores entre o sexo feminino. Quanto à cor da pele, verifica-se o mesmo resultado encontrado para o período anterior. A média de permanência variou de 1,0 a 4,2 dias, com as exceções de Acre (18,7 dias) e Pernambuco (22,6 dias). Oito pacientes vieram a óbito.

No Gráfico 1, pode-se observar a evolução temporal das internações por tentativa de suicídio em crianças para o período 2006-2017 segundo o sexo. A avaliação da série temporal mostra taxas maiores para os meninos, e queda, ao longo dos anos, para ambos os sexos. Verifica-se que a taxa atingiu seu máximo em 2008, quando chegou a aproximadamente 1,9 internação/100.000 habitantes entre os meninos.

No Gráfico 2 é apresentada a evolução temporal das internações por tentativa de suicídio segundo regiões do país. A região Norte se destaca, chegando a registrar, em 2008, taxa de cerca de 7 internações/100.000 habitantes. Observa-se redução das taxas ao longo dos anos, com indicação de aumento em 2015 nas regiões Sudeste e Centro-Oeste.

Com relação aos dados de mortalidade (SIM), verificou-se que 58 óbitos de crianças brasileiras foram decorrentes de lesões autoprovocadas intencionalmente (X60-X84 da CID-10) no período de 2006 a 2017. A despeito desse preenchimento, identificou-se mais casos na variável provável circunstância do óbito não natural: 11 classificados como acidentes, 34 como suicídios e 13 como ignorados. Visando um maior rigor na análise, selecionou-se apenas os óbitos cuja circunstância foi reiterada como suicídio, cuja distribuição segundo sexo e demais variáveis é apresentada na Tabela 4. 


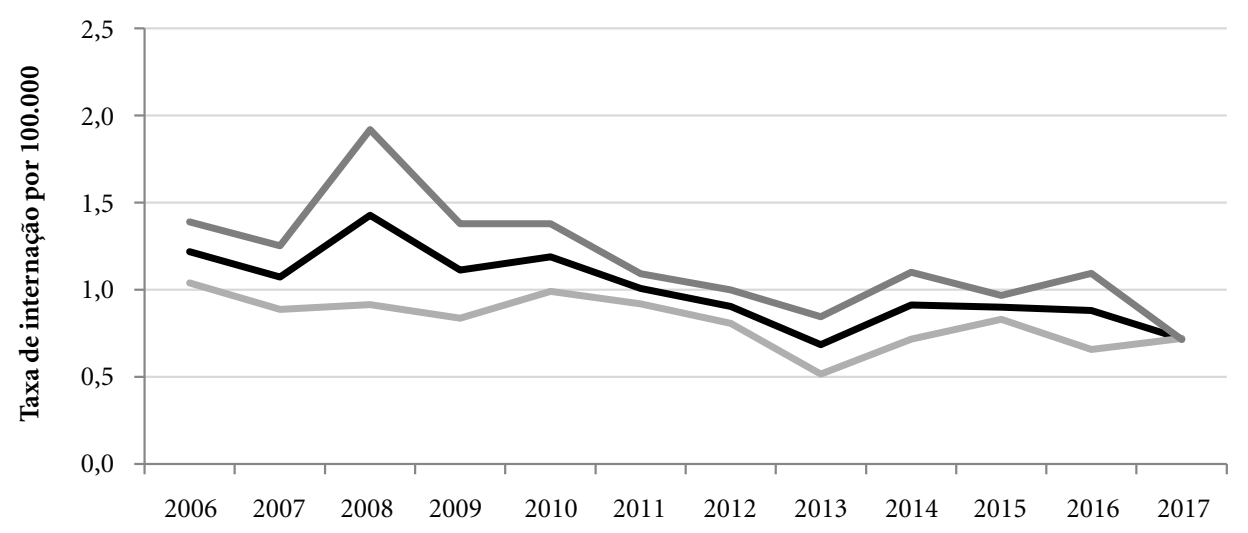

Ano

$$
\longrightarrow \text { tx geral } \rightleftharpoons \text { tx feminina } \rightleftharpoons \text { masculina }
$$

Gráfico 1. Evolução temporal das taxas de internações por tentativa de suicídio em crianças de cinco a nove anos para o período 2006-2017 segundo gênero

Fonte: Sistema de Informações Hospitalares (SIH), Ministério da Saúde.

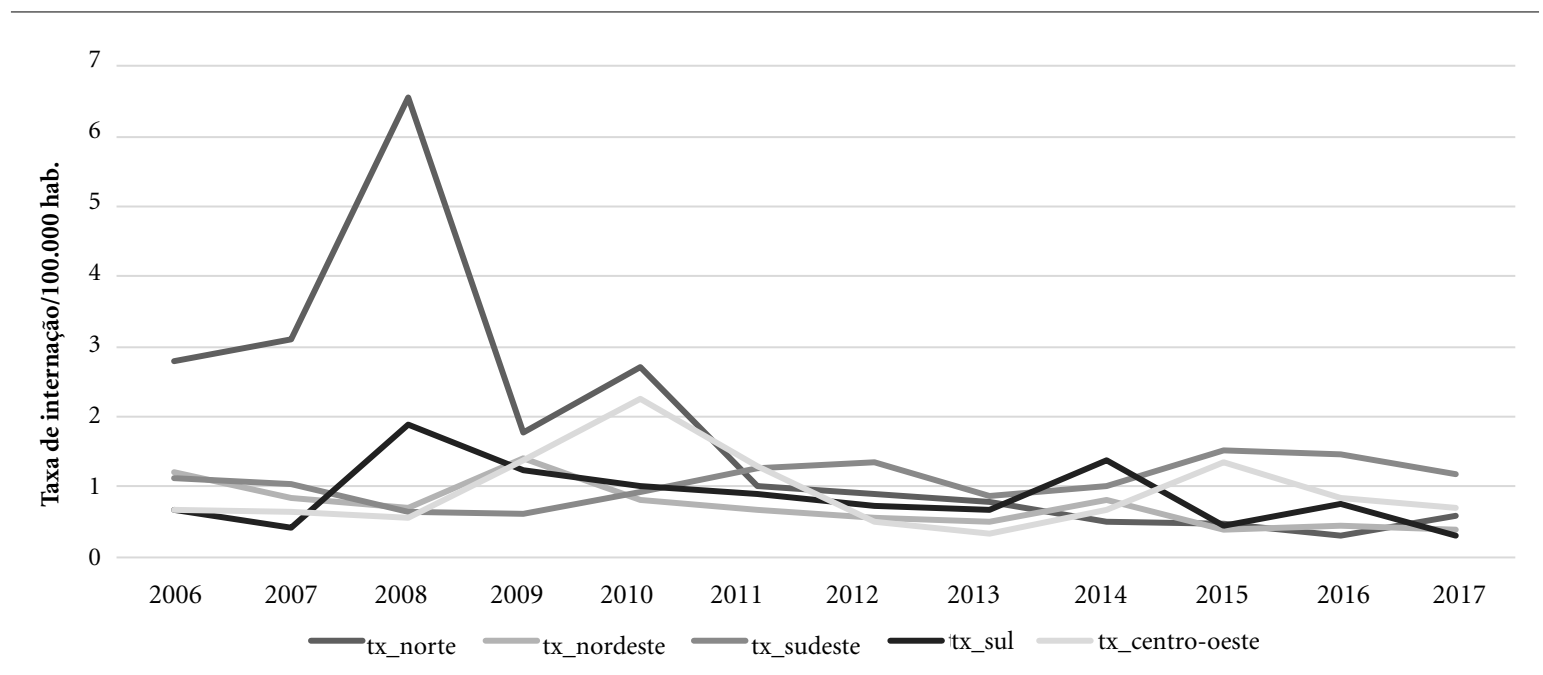

Gráfico 2. Evolução temporal das taxas de internação por tentativas de suicídio em crianças de cinco a nove anos para o período 2006-2017 segundo região

Fonte: Sistema de Informações Hospitalares (SIH), Ministério da Saúde.

Pode-se observar que 29 óbitos foram em indivíduos do sexo masculino, e cinco do sexo feminino (Tabela 4). Quanto à distribuição temporal, observa-se uma redução a partir de 2012, e novamente um aumento nos anos de 2016 e 2017. A maioria dos óbitos foi de crianças de nove anos de idade (21 óbitos), iniciando-se o registro aos sete anos de idade, com maioria de 
Tabela 4. Óbitos por suicídio na população brasileira de cinco a nove anos, 2006-2017.

\begin{tabular}{|c|c|c|c|c|c|c|}
\hline \multirow{3}{*}{ Variável } & \multicolumn{4}{|c|}{ Sexo } & \multirow{2}{*}{\multicolumn{2}{|c|}{ Total }} \\
\hline & \multicolumn{2}{|c|}{ Masculino } & \multicolumn{2}{|c|}{ Feminino } & & \\
\hline & $\mathbf{n}$ & $\%$ & $\mathbf{n}$ & $\%$ & $\mathbf{n}$ & $\%$ \\
\hline \multicolumn{7}{|l|}{ Ano de ocorrência do óbito } \\
\hline 2006 & 5 & 17,2 & 2 & 40,0 & 7 & 20,6 \\
\hline 2007 & 1 & 3,4 & 0 & 0,0 & 1 & 2,9 \\
\hline 2008 & 4 & 13,8 & 0 & 0,0 & 4 & 11,8 \\
\hline 2009 & 5 & 17,2 & 0 & 0,0 & 5 & 14,7 \\
\hline 2010 & 2 & 6,9 & 1 & 20,0 & 3 & 8,8 \\
\hline 2012 & 1 & 3,4 & 1 & 20,0 & 2 & 5,9 \\
\hline 2013 & 0 & 0,0 & 1 & 20,0 & 1 & 2,9 \\
\hline 2014 & 1 & 3,4 & 0 & 0,0 & 1 & 2,9 \\
\hline 2015 & 2 & 6,9 & 0 & 0,0 & 2 & 5,9 \\
\hline 2016 & 3 & 10,3 & 0 & 0,0 & 3 & 8,8 \\
\hline 2017 & 5 & 17,2 & 0 & 0,0 & 5 & 14,7 \\
\hline \multicolumn{7}{|l|}{ Idade em anos } \\
\hline 7,00 & 5 & 17,2 & 0 & 0,0 & 5 & 14,7 \\
\hline 8,00 & 7 & 24,1 & 1 & 20,0 & 8 & 23,5 \\
\hline 9,00 & 17 & 58,6 & 4 & 80,0 & 21 & 61,8 \\
\hline \multicolumn{7}{|l|}{ Cor da pele } \\
\hline Branca & 10 & 34,5 & 3 & 60,0 & 13 & 38,2 \\
\hline Preta & 1 & 3,4 & 0 & 0,0 & 1 & 2,9 \\
\hline Amarela & 0 & 0,0 & 0 & 0,0 & 0 & 0,0 \\
\hline Parda & 12 & 41,4 & 0 & 0,0 & 12 & 35,3 \\
\hline Indígena & 4 & 13,8 & 2 & 40,0 & 6 & 17,6 \\
\hline Ignorado & 2 & 6,9 & 0 & 0,0 & 2 & 5,9 \\
\hline \multicolumn{7}{|l|}{ Escolaridade } \\
\hline Nenhum & 0 & 0,0 & 0 & 0,0 & 0 & 0,0 \\
\hline 1 a 3 anos & 12 & 41,4 & 1 & 20,0 & 13 & 38,2 \\
\hline 4 a 7 anos & 4 & 13,8 & 2 & 40,0 & 6 & 17,6 \\
\hline 8 a 11 anos & 0 & 0,0 & 0 & 0,0 & 0 & 0,0 \\
\hline 12 ou mais & 0 & 0,0 & 0 & 0,0 & 0 & 0,0 \\
\hline Ignorado & 13 & 44,8 & 2 & 40,0 & 15 & 44,1 \\
\hline Local de ocorrência & & & & & & 0,0 \\
\hline Hospital & 12 & 41,4 & 1 & 20,0 & 13 & 38,2 \\
\hline Outro estabelecimento de saúde & 0 & 0,0 & 0 & 0,0 & 0 & 0,0 \\
\hline Domicílio & 15 & 51,7 & 4 & 80,0 & 19 & 55,9 \\
\hline Via pública & 1 & 3,4 & 0 & 0,0 & 1 & 2,9 \\
\hline Outros & 1 & 3,4 & 0 & 0,0 & 1 & 2,9 \\
\hline Ignorado & 0 & 0,0 & 0 & 0,0 & 0 & 0,0 \\
\hline
\end{tabular}

cor da pele branca (para ambos os sexos) e parda (apenas entre os meninos). É interessante notar que, dos cinco óbitos por suicídio em meninas, duas eram indígenas. O domicílio foi o local de ocorrência de 55,9\% dos óbitos. Quanto à região, destacam-se 11 óbitos na região Norte e 10 na região Nordeste. Discriminando por UF, verificase que, para os meninos, destacam-se Amazonas,
Pará, Ceará, Rio Grande do Sul e Mato Grosso do Sul. Entre a meninas, ocorreu um óbito apenas em cada uma das seguintes UFs: Acre, Amazonas, Maranhão, Minas Gerais e Santa Catarina. O enforcamento foi o meio mais utilizado pelas crianças para se matar. A fonte da informação não foi preenchida em 12 registros e também em 12 casos baseou-se no boletim de ocorrência. 
Tabela 4. Óbitos por suicídio na população brasileira de cinco a nove anos, 2006-2017.

\begin{tabular}{|c|c|c|c|c|c|c|}
\hline \multirow{3}{*}{ Variável } & \multicolumn{4}{|c|}{ Sexo } & \multirow{2}{*}{\multicolumn{2}{|c|}{ Total }} \\
\hline & \multicolumn{2}{|c|}{ Masculino } & \multicolumn{2}{|c|}{ Feminino } & & \\
\hline & $\mathbf{n}$ & $\%$ & $\mathbf{n}$ & $\%$ & $\mathbf{n}$ & $\%$ \\
\hline \multicolumn{7}{|l|}{ UF de ocorrência } \\
\hline Acre & 1 & 3,4 & 1 & 20,0 & 2 & 5,9 \\
\hline Amazonas & 4 & 13,8 & 1 & 20,0 & 5 & 14,7 \\
\hline Pará & 4 & 13,8 & 0 & 0,0 & 4 & 11,8 \\
\hline Maranhão & 1 & 3,4 & 1 & 20,0 & 2 & 5,9 \\
\hline Piauí & 1 & 3,4 & 0 & 0,0 & 1 & 2,9 \\
\hline Ceará & 3 & 10,3 & 0 & 0,0 & 3 & 8,8 \\
\hline Alagoas & 2 & 6,9 & 0 & 0,0 & 2 & 5,9 \\
\hline Bahia & 2 & 6,9 & 0 & 0,0 & 2 & 5,9 \\
\hline Minas Gerais & 0 & 0,0 & 1 & 20,0 & 1 & 2,9 \\
\hline São Paulo & 2 & 6,9 & 0 & 0,0 & 2 & 5,9 \\
\hline Paraná & 2 & 6,9 & 0 & 0,0 & 2 & 5,9 \\
\hline Santa Catarina & 0 & 0,0 & 1 & 20,0 & 1 & 2,9 \\
\hline Rio Grande do Sul & 3 & 10,3 & 0 & 0,0 & 3 & 8,8 \\
\hline Mato Grosso do Sul & 3 & 10,3 & 0 & 0,0 & 3 & 8,8 \\
\hline Mato Grosso & 1 & 3,4 & 0 & 0,0 & 1 & 2,9 \\
\hline \multicolumn{7}{|l|}{ Região de ocorrência } \\
\hline Norte & 9 & 31,0 & 2 & 40,0 & 11 & 32,4 \\
\hline Nordeste & 9 & 31,0 & 1 & 20,0 & 10 & 29,4 \\
\hline Sudeste & 2 & 6,9 & 1 & 20,0 & 3 & 8,8 \\
\hline Sul & 5 & 17,2 & 1 & 20,0 & 6 & 17,6 \\
\hline Centro oeste & 4 & 13,8 & 0 & 0,0 & 4 & 11,8 \\
\hline \multicolumn{7}{|l|}{ Meio empregado (Categoria CID-10) } \\
\hline $\begin{array}{l}\text { Autointoxicação por e exposição, intencional, a outras } \\
\text { drogas, medicamentos e substâncias biológicas e às } \\
\text { não especificadas }\end{array}$ & 1 & 3,4 & 0 & 0,0 & 1 & 2,9 \\
\hline $\begin{array}{l}\text { Autointoxicação por e exposição, intencional, a } \\
\text { pesticidas }\end{array}$ & 0 & 0,0 & 1 & 20,0 & 1 & 2,9 \\
\hline $\begin{array}{l}\text { Lesão autoprovocada intencionalmente pela fumaça, } \\
\text { pelo fogo e por chamas }\end{array}$ & 1 & 3,4 & 0 & 0,0 & 1 & 2,9 \\
\hline $\begin{array}{l}\text { Lesão autoprovocada intencionalmente por disparo } \\
\text { de outra arma de fogo e de arma de fogo não } \\
\text { especificada }\end{array}$ & 1 & 3,4 & 0 & 0,0 & 1 & 2,9 \\
\hline $\begin{array}{l}\text { Lesão autoprovocada intencionalmente por } \\
\text { enforcamento, estrangulamento e sufocação }\end{array}$ & 26 & 89,7 & 4 & 80,0 & 30 & 88,2 \\
\hline \multicolumn{7}{|l|}{ Fonte da informação } \\
\hline Boletim de ocorrência & 11 & 37,9 & 1 & 20,0 & 12 & 35,3 \\
\hline Hospital & 7 & 24,1 & 0 & 0,0 & 7 & 20,6 \\
\hline Família & 0 & 0,0 & 2 & 40,0 & 2 & 5,9 \\
\hline Outra & 1 & 3,4 & 0 & 0,0 & 1 & 2,9 \\
\hline Ignorado & 10 & 34,5 & 2 & 40,0 & 12 & 35,3 \\
\hline
\end{tabular}

Fonte: Sistema de Informação sobre Mortalidade (SIM), Ministério da Saúde.

\section{Discussão}

A natureza descritiva apresentada neste artigo se mostra a única forma de aproximação a uma temática ainda pouco investigada no país, caracterizando-se por seu ineditismo e por tirar da invisibilidade um tema ainda tão pouco conhecido. Os achados encontrados precisam ser disseminados e para servirem como sinal de alerta para profissionais de saúde e da educação, dada a gravidade do problema e sua subnotificação. Em todo o mundo, quase não há números ofi- 
ciais aobre o comportamento suicida na infância, dificultando a comparação com outros países. Nos Estados Unidos, o Centro Nacional para Prevenção e Controle de Lesões ${ }^{14}$ mostrou que, em 2003, o suicídio nessa faixa etária foi responsável por 68 óbitos de crianças menores de 12 anos, a maioria por sufocamento (incluindo enforcamento ou estrangulamento), e um pouco menos por arma de fogo.

Fazendo um paralelo com o suicídio na fase da adolescência, enquanto o índice cai em todo o mundo, entre adolescentes brasileiros, de 2006 e 2015 , aumentou $24 \%$ entre aqueles que vivem em algumas grandes cidades do Brasil, com destaque para as capitais do Sudeste ${ }^{28}$. Esse cenário parece dialogar com uma possível tendência de crescimento das taxas de internação por lesão autoprovocada em crianças nas regiões Sudeste e Centro-Oeste a partir de 2013. Também se nota maior número de notificações por essa causa entre 2015 e 2017. Entre as explicações para o incremento do comportamento suicida em qualquer faixa etária, estão: as características locais, as mudanças sociais no país e a falta de políticas públicas de enfrentamento ao fenômeno. Asevedo e colaboradores ${ }^{29}$ explicam que fatores locais, como políticas de proteção ao desemprego, apoio social e distribuição da população por sexo e por áreas urbanas ou rurais, são importantes moderadores para o comportamento suicida. Para as crianças, tais aspectos são muito mediados pelo impacto que terão em suas famílias, em seus cuidadores e em sua vida cotidiana.

Apesar da melhoria dos sistemas de informação de saúde no Brasil nos últimos anos, especialmente nas notificações por violências, os dados revelam a incipiência da magnitude dos comportamentos suicidas em crianças que chegam aos serviços de saúde, tendo como contraponto fundamental a fragilidade dos registros. Um número significativo de casos pode nem ter sido categorizado como a causa estudada, pois como são crianças os atos contra a própria vida são negados ou até escondidos pela família, diante de sentimentos de culpa ou vergonha. Barreiras socioculturais, morais e religiosas dificultam a precisão na identificação do problema, o que interfere na qualidade da informação, mascarando muito o problema ${ }^{30}$.

Interessante perceber que alguns perfis são muito semelhantes ao encontrado entre adolescentes com comportamento suicida, o que mostra certa coerência no contínuo desse comportamento da infância até a adolescência. Tanto para as notificações de tentativas quanto para os óbitos prevalece a maior ocorrência entre as crianças mais velhas, com cor de pele branca para ambos os sexos e preta/parda entre os meninos do Norte e Nordeste do país, e o domicílio como o local de maior ocorrência ${ }^{4,5}$. Joe, Banks e Belue $\mathrm{e}^{25}$ explicam que há poucas conclusões para explicar as diferenças étnico-raciais do suicídio, apesar de estudos recentes ${ }^{31,32}$ mostrarem taxas mais altas em crianças e adolescentes negros. Da mesma maneira, vale destacar que a liderança do problema nas regiões Norte e Centro-Oeste pode estar atrelada, entre outras questões, à sua maior ocorrência entre os povos indígenas, o que tem sido explicado pela perda de valores culturais tradicionais, o elevado consumo de álcool nas famílias, o alto índice de depressão, a falta de perspectiva de vida e o desemprego ${ }^{8}$. Nos dados oficiais apresentados, a presença das crianças indígenas entre cinco e nove anos se manifesta de forma tênue, diferenciando-se de outro estudo, para a faixa dos 10-14 anos, que evidencia que a taxa de mortalidade por suicídio entre crianças indígenas brasileiras é 18,5 vezes maior do que a observada entre as não indígenas ${ }^{33}$. Nessa linha de pensamento, Jaen-Varas ${ }^{28}$, ao estudar adolescentes, também alerta que a maior parte da variação das taxas de suicídio são relacionadas aos fatores de risco dos locais de moradia e das cidades.

A literatura indica que, assim como ocorre na adolescência, os meninos morrem mais por suicídio e as meninas apresentam mais notificações por lesão autoprovocada. Esses achados dialogam com o meio escolhido por crianças para morrer: no suicídio, em epecial entre os meninos, o sufocamento é o meio mais utilizado, enquanto, de acordo com as notificações, as meninas utilizam meios menos lesivos, como a autointoxicação por ingestão de medicamentos e a lesão autoprovocada intencionalmente por objeto cortante ou penetrante ${ }^{28,30-35}$. Na contramão da literatura, é interessante notar que os meninos lideram as internações por tentativas de suicídio, o que pode sugerir a escolha por métodos mais lesivos e que necessitam de maior atenção pelo serviço de saúde. Os meios utilizados por crianças nas tentativas e no suicídio mostram a noção que elas têm do que pode levar à morte.

Os achados encontrados dialogam com as tradicionais questões de gênero relacionadas ao tema, que apontam que os meninos buscam métodos mais violentos e letais nas tentativas de suicídio, o que parece muito baseado na socialização masculina, que valoriza a competitividade e agressividade, a maior exposição a situações de risco, além da maior vulnerabilidade de cuidados 
e afeto a eles dirigidos. Por sua vez, as meninas costumam receber mais apoio e, quando deprimidas, procuram mais ajuda ${ }^{5}$.

Entre as limitações do estudo, é importante levar em consideração as fragilidades das estatísticas dos sistemas de informação de saúde. Primeiro, os dados existentes investigam uma fração do problema, deixando de abordar a ideação e o planejamento suicida. Outro fator é que o sistema VIVA ainda não tem cobertura nacional e é muito recente em relação às lesões autoprovocadas. Sabe-se que os casos que chegam aos serviços de saúde são por si só mais graves. Na população geral, estima-se que, para cada três tentativas, apenas uma é atendida em serviço médico de urgência. $\mathrm{O}$ estigma em relação ao comportamento suicida e o medo da criminalização do ato são os motivos principais para a ausência do registro do episódio nos sistemas de saúde ${ }^{36-38}$. Em segundo lugar, há subnotificação dos dados por erros de classificação pelo profissional de saúde ${ }^{30}$. Além do mais, os dados praticamente só abarcam a rede pública de saúde.

Por fim, este estudo mostra o cenário do comportamento suicida na infância no Brasil nos últimos anos, que se assemelha muito ao do adolescente. A evidência de que qualquer comportamento suicida na infância está fortemente associado às tentativas ou ao suicídio consumado na adolescência e na vida adulta é uma das principais indicações da necessidade de prevenção desse comportamento na primeira década de vida. Assim, é urgente que a rede pública de saúde esteja qualificada para atender tais casos desde a primeira etapa da vida, quando muitas vezes é difícil delimitar com clareza a intencionalidade do ato, mas em que a escuta qualificada, a internação (se necessária) e o monitoramento precisam ser nortes para a atenção. Além disso, é preciso dar efetividade à Política Nacional de Prevenção da Automutilação e do Suicídio ${ }^{39}$ e desenvolver protocolos oficiais que incluam inquérito policial, autópsia psicológica e pósvenção ${ }^{35}$. Empregar um modelo preventivo para identificar crianças que possam estar em risco é mais eficaz para a redução da taxa de suicídio do que se concentrar na limitação do acesso aos métodos. Assim, compreender e identificar fatores que colocam em risco pré-púberes é uma estratégia de prevenção a longo prazo, até a vida adulta.

\section{Colaboradores}

JQ Avanci trabalhou na concepção e na redação, LW Pinto trabalhou no delineamento metodológico e na análise dos dados, SG Assis trabalhou na concepção e na redação final. 


\section{Referências}

1. Kuczynski E. Suicídio na infância e adolescência. Psicologia USP 2014; 25(3):246-52.

2. Pedroso JD. Suicídio na Infância. Revista AMIRGS 1986; 30(4):289-293.

3. Fensterseifer L, Werlang BS. Suicídio na infância: será a perda da inocência? Psicologia Argumento 2003; 21(35):39-46.

4. Organização Mundial de Saúde (OMS). Relatório mundial sobre violência e saúde. Genebra: Organização Mundial de Saúde; 2002.

5. Botega NJ. Crise suicida: avaliação e manejo. Porto Alegre: Artmed; 2015.

6. Organização Mundial da Saúde (OMS). Classificação Estatística Internacional de Doenças e Problemas Relacionados à Saúde. $10^{\circ}$ Revisão. São Paulo: Faculdade de Saúde Pública da Universidade de São Paulo; 2009.

7. Mishara B. How the media influences children's conceptions of suicide. Crisis 2003; 24(3):128-30.

8. Beautrais A, Fergusson DM. Indigenous suicide in New Zealand. Arch Suicide Res 2006; 10(2):159-168.

9. World Health Organization/Department of Mental Health. Figures and facts about suicide. Geneva: World Health Organization; 1999.

10. Kerr GR, Ramsey DJ, Spears W. Searching for preventable causes of child mortality in Texas: trends in the major causes from 1987 through 1996. Tex Med 1999; 95(7):56-64.

11. Goldman S, Beardslee WR. Suicide in children and adolescents. In: Jacobs DG, editor. The Harvard Medical School guide to suicide assessment and intervention. San Francisco: Jossey-Bass;1999. p.417-442.

12. Lee CJ, Collins KA, Burgess SE. Suicide under the age of eighteen. Am J Forensic Med Pathol 1999; 20:27-30.

13. Centers for Disease Control and Prevention. Leading Causes of Death Reports, National and Regional, 1999-2015; 2016. acessado 2017 Ago 3. Available from: http://webappa.cdc.gov/sasweb/ncipc/leadcaus10 _us.html

14. Centers for Disease Control and Prevention. Fatal injury reports, national and regional, 1999-2014; 2016. [acessado 2017 Ago 3]. Available from: http://webappa.cdc.gov/sasweb/ncipc/mortrate10_us.html

15. Heron M. Deaths: leading Causes for 2013. Natl Vital Stat Rep 2016; 65(2):1-95.

16. Organização Mundial de Saúde (OMS). Relatório sobre a saúde mental no mundo. Genebra: OMS; 2014.

17. Bridge JA, Goldstein TR, Brent DA. Adolescent suicide and suicidal behavior. J Child Psychol Psychiatry 2006; 47(3-4):372-394.

18. Lewinsohn PM, Rohde P, Seeley JR. Psychosocial risk factors for future adolescent suicide attempts. J Consul Clin Psychol 1994; 62(2):297-305.

19. Plener PL, Schumacher TS, Munz LM, Groschwitz RC. The longitudinal course of non-suicidal self-injury and deliberate self-harm: a systematic review of the literature. Borderline Personal Disord Emot Dysregul 2015; 2:2. doi: 10.1186/s40479-014-0024-3.

20. Kloos AL, Collins R, Weller RA, Weller EB. Suicide in preadolescents: who is at risk? Curr Psychiatr 2007; 9(2):89-93.
21. Brent D. Commentary: A time to reap and a time to sow: reducing the adolescent suicide rate now and in the future: commentary on Cha et al. Journal Child Psychol Psychiatr 2018; 59(4):483-485.

22. Kosky R. Childhood suicidal behaviour. Journal Child Psychol and Psychiatr 1983; 24:457-468.

23. Sousa GS, Santos MSP, Silva ATP, Perrelli JGA, Sougey EB. Revisão de literatura sobre suicídio na infância. Cien Saude Colet 2017; 22(9):3099-3110.

24. Rodway C, Tham SG, Ibrahim S, Turnbull P, Windfuhr K, Shaw J, Kapur N, Appleby L. Suicide in children and young people in England: a consecutive case series. Lancet Psychiatry 2016; 3(8):751-759.

25. Joe S, Banks A, Belue R. Suicide risk among urban children. Children and Youth Services Review 2016, 68: 73-79.

26. Brasil. Ministério da Saúde (MS). Secretaria de Vigilância em Saúde. Departamento de Vigilância de Doenças e Agravos não Transmissíveis e Promoção da Saúde. Sistema de Vigilância de Violências e Acidentes (Viva): 2009, 2010 e 2011. Brasília: MS; 2013.

27. Pinto LW, Assis SG. Estudo descritivo das tentativas de suicídio na população idosa brasileira, 2000-2014. Cien Saude Colet 2015; 20(6):1681-1692.

28. Jaen-Varas D, Mari JJ, Asevedo E, Borschmann R, Diniz E, Ziebold C, Gadelha A. The association between adolescent suicide rates and socioeconomic indicators in Brazil: a 10-year retrospective ecological study. Braz J Psychiatry 2019; 41(5):389-395.

29. Asevedo E, Ziebold C, Diniz E, Gadelha A, Mari J. Ten-year evolution of suicide rates and economic indicators in large Brazilian urban centers. Curr Opin Psychiatry 2018, 31(3):265-271.

30. Bahia CA, Avanci JQ, Pinto LW, Minayo MCS. Lesão autoprovocada em todos os ciclos de vida: perfil das vítimas em serviços de urgência e emergência de capitais do Brasil. Cien Saude Colet 2017; 22(9):2841-2850

31. Bridge JA, Asti L, Horowitz LM, Greenhouse JB, Fontanella CA, Sheftall AH, Kelleher KJ, Campo JV. Suicide trends among elementary school-aged children in the united states from 1993 to 2012. JAMA Pediatr 2015; 169(7):673-677.

32. Brasil. Ministério da Saúde (MS). Secretaria de Gestão Estratégica e Participativa. Departamento de Apoio à Gestão Participativa e ao Controle Social. Óbitos por suicídio entre adolescentes e jovens negros 2012 a 2016. Universidade de Brasília, Observatório de Saúde de Populações em Vulnerabilidade. Brasília: MS; 2018.

33. Silva, MLP. Mortalidade por suicídio entre crianças indígenas no Brasil. Cad Saude Publica 2019; 35(supl. 3):1-12.

34. Machado DB, Santos DN. Suicídio no Brasil, de 2000 a 2012. J Bras Psiquiatr 2015; 64(1):45-54.

35. Beautrais AL. Suicides and serious suicide attempts: two populations or one? Psychol Med 2001; 31(5):837845

36. Botega NJ. Comportamento suicida: conhecer pra prevenir. São Paulo: Associação Brasileira de Psiquiatria; 2009.

37. Nock MK, Borges G, Bromet EJ, Cha CB, Kessler RC, Lee S. Suicide and suicidal behavior. Epidemiol Rev 2008; 30(1):133-154. 
38. Bostwick JM. Do SSRIs cause suicide in children? The evidence is underwhelming. J Clin Psychol 2006; 62(2):235-241.

39. Brasil. Lei no 13.819 , de 26 de abril de 2019. Institui a Política Nacional de Prevenção da Automutilação e do Suicídio, a ser implementada pela União, em cooperação com os Estados, o Distrito Federal e os Municípios; e altera a Lei no 9.656, de 3 de junho de 1998. Vigência. Brasília: Diário Oficial da União 2019; 26 abr.

Artigo apresentado em 08/09/2019

Aprovado em 09/01/2020

Versão final apresentada em 11/01/2020

Editores-chefes: Romeu Gomes, Antônio Augusto Moura da Silva 